\title{
Methionine and Cystine in Puerto Rican Institutional Dishes
}

\author{
Conrado F. Asenjo and Evangelina Recio ${ }^{1}$
}

\section{INTRODUCTION AND OBJECTIVE}

The basic Puerto Rican diet is known to be low in methionine and cystine. It consists principally of starchy vegetables, polished rice, beans (red kidney and navy beans principally), small portions of codfish and still less of other minor constituents.

Cook, Axtmayer, and Dalmau (1) ${ }^{2}$ reported that some growth improvement took place when rats were fed this diet supplemented with cystine. Axtmayer (2) showed that the growth of rats fed a rice-and-bean diet was dramatically improved when supplemented with small amounts of methionine. Asenjo and Goyco ( 3 ) confirmed this observation. Recently, Goyco (4) reported significant improvement in the nitrogen balance of human volunteers kept exclusively on the Puerto Rican basic diet when this was supplemented with methionine.

The purpose of the present investigation was to determine the methionine and cystine contents of dishes commonly served at the Cafeteria of the School of Medicine of the University of Puerto Rico.

\section{MATERIALS AND METHODS}

All the dishes studied were cooked at the Cafeteria of the School of Medicine under the care of graduate dietitians according to accepted methods. At least three different samples of each dish were assayed. All determinations were made in duplicate.

Moisture was determined by heating the samples at $100^{\circ} \mathrm{C}$. to constant weight. Nitrogen was determined according to the official semimicro Kjeldahl method of the Official Methods of Analysis of the AOAC (5), although using a smaller sample than the one recommended, and selenized Hengar granules as catalyst. Methionine was assayed by the microbiological method of Stokes, et al. (6), using Streptococcus fecalis (S. Lactis R.).

1 Professor and Head, Department of Biochemistry and Nutrition, and Research Assistant in Biochemistry and Nutrition, respectively, at the School of Medicine, School of Tropical Medicine, University of Puerto Rico, San Juan 22, P.R. A cooperative project with the Agricultural Experiment Station of the University of Puerto Rico. The authors wish to thank Misses Margarita Saldaña and Yolanda Suárez, dietitians at the School Cafeteria, for providing the servings of each dish analyzed and also the recipes.

${ }^{2}$ Italic numbers in parentheses refer to Literature Cited, p. 359. 
Cystine was assayed following essentially the microbiological method of Barton-Wright (7), although the sample was hydrolyzed in the autoclave at $15 \mathrm{lb}$. pressure for a period of 2 hours only, as recommended by Horn and Blum (8). Difco's dehydrated medium for cystine was used as the basal medium and Leuconostoc mesenteroides $\mathrm{P} 60$, as the test organism.

All the organisms used in these assays were obtained from the American Type Culture Collections in Washington, D.C.

\section{RESULTS AND DISCUSSION}

The results are reported in table 1 . In the following discussion all values for methionine and cystine are reported on the basis of the dish as served.

The dishes composed mainly of animal proteins were the ones with the highest content of methionine and cystine. Thus the highest methionine content was exhibited by stuffed meat and stewed goat, 876.1 and 829.0 $\mathrm{mg}$. per $100 \mathrm{gm}$. as served, respectively. Both of these dishes were also high in cystine, 191.0 and $125.8 \mathrm{mg}$. per $100 \mathrm{gm}$., respectively. The meat dish with the lowest methionine and cystine content was entrails of chicken, $251.9 \mathrm{mg}$. of methionine per $100 \mathrm{gm}$. and $55.7 \mathrm{mg}$. of cystine per $100 \mathrm{gm}$. The meat dish with the highest cystine content was roasted thigh of pork with $291.0 \mathrm{mg}$. per $100 \mathrm{gm}$. This dish was also high in methionine with $778.8 \mathrm{mg}$. per $100 \mathrm{gm}$. The only fish dish tested, breaded perch, had a high methionine content but a rather low cystine when compared with meat dishes.

The dishes with the lowest content of both methionine and cystine are those composed exclusively of vegetables. Boiled arracacha was the dish with the lowest content of methionine, $5.5 \mathrm{mg}$. per $100 \mathrm{gm}$., and cystine $0.9 \mathrm{mg}$. per $100 \mathrm{gm}$. It is interesting to note that root-crop dishes in general had a higher content of cystine than the other vegetable dishes. Boiled yellow tanier had the highest cystine values, $21.6 \mathrm{mg}$. per $100 \mathrm{gm}$., among the vegetable dishes.

Among the socalled cereal foods the different breads assayed showed the highest content of methionine and cystine. Methionine was over $130 \mathrm{mg}$. per $100 \mathrm{gm}$. and cystine over $120 \mathrm{mg}$. per $100 \mathrm{gm}$. As bread is eaten in appreciable quantity it contributes a substantial amount of these two amino acids to the daily diet.

Beans, in general, were relatively low in methionine and cystine, ranging from 44.7 to $36.8 \mathrm{mg}$. per $100 \mathrm{gm}$. and from 13.8 to $7.4 \mathrm{mg}$. per $100 \mathrm{gm}$., respectively.

The majority of the desserts ranged in methionine between 85.7 and $24.0 \mathrm{mg}$. per $100 \mathrm{gm}$. and in cystine between 35.8 and $13.7 \mathrm{mg}$. per $100 \mathrm{gm}$. Two desserts that rated well above the rest, probably because they con- 
TABLE 1.-Methionine and cystine in Puerto Rican institutional dishes

\begin{tabular}{|c|c|c|c|c|c|c|c|}
\hline Name of dish in English & Name of dish in Spanish & Method of cooking & $\begin{array}{c}\text { Samples } \\
\text { tested }\end{array}$ & Moisture & $\begin{array}{c}\text { Protein, } \\
\text { wet } \\
\text { basis }\end{array}$ & $\begin{array}{l}\text { Methionine per } 100 \\
\text { gm. as served: } \\
\text { Average maximum } \\
\text { and minimum in } \\
\text { parentheses }\end{array}$ & $\begin{array}{l}\text { Cystine per } 100 \mathrm{gm} \text {. } \\
\text { as served: Average } \\
\text { maximum and } \\
\text { minimum in } \\
\text { parentheses }\end{array}$ \\
\hline & & & Number & Percent & Percent & sfilligrams & Milligrams \\
\hline \multicolumn{8}{|l|}{ Vegetables: } \\
\hline Arracacha & Apio & Boiled & 3 & 69.6 & 1.0 & $\begin{array}{c}5.5 \\
(8.5-3.6)\end{array}$ & $\begin{array}{c}0.9 \\
(1.1-0.8)\end{array}$ \\
\hline Banana, green & Guineo verde & do. & 3 & 74.6 & 1.2 & $\begin{array}{c}11.4 \\
(13.5-9.1)\end{array}$ & $\begin{array}{c}2.5 \\
(3.1-2.1)\end{array}$ \\
\hline Cabbage & Repollo & do. & 3 & 85.1 & 1.4 & $\begin{array}{c}16.5 \\
(21.0-14.0)\end{array}$ & $\begin{array}{c}4.0 \\
(4.1-3.8)\end{array}$ \\
\hline Carrots, boiled & Zanahorias hervidas & Boiled; butter added & 3 & 86.3 & 0.8 & $\begin{array}{c}8.5 \\
(10.3-7.3)\end{array}$ & $\begin{array}{c}1.0 \\
(1.0-1.0)\end{array}$ \\
\hline Carrot and raisin salad & $\begin{array}{l}\text { Ensalada de zanahorias y } \\
\text { pasas }\end{array}$ & Raw & 3 & 71.1 & 1.4 & $\begin{array}{c}7.5 \\
(11.0-5.8)\end{array}$ & $\begin{array}{c}2.1 \\
(2.3-2.0)\end{array}$ \\
\hline Corn, cream style & Maíz a la crema & Cream style & 3 & 76.1 & 2.1 & $\begin{array}{c}52.6 \\
(56.4-46.4)\end{array}$ & $\begin{array}{c}6.3 \\
(6.7-5.7)\end{array}$ \\
\hline Corn and lima beans & Maíz y habas & Boiled; butter added & 3 & 69.0 & 4.4 & $\begin{array}{c}68.5 \\
(73.2-62.9)\end{array}$ & $\begin{array}{c}13.8 \\
(15.7-11.6)\end{array}$ \\
\hline Corn, whole kernel & Maíz en grano & do. & 3 & 65.5 & 3.0 & $\begin{array}{c}71.8 \\
(80.7-65.2)\end{array}$ & $\begin{array}{c}13.2 \\
(13.9-12.5)\end{array}$ \\
\hline Mixed vegetables & Vegetales mixtos & do. & 3 & 82.3 & 2.9 & $\begin{array}{c}41.6 \\
(45.3-38.2)\end{array}$ & $\begin{array}{c}12.8 \\
(14.9-10.7)\end{array}$ \\
\hline Peas and carrots & Guisantes y zanahorias & do. & 3 & 79.9 & 3.4 & $\begin{array}{c}29.9 \\
(38.4-19.9)\end{array}$ & $\begin{array}{c}8.4 \\
(8.7-8.0)\end{array}$ \\
\hline Plantain, green & Plátano verde & Boiled & 3 & 64.4 & 0.9 & $\begin{array}{c}8.9 \\
(9.3-8.2)\end{array}$ & $\begin{array}{c}2.0 \\
(2.2-1.7)\end{array}$ \\
\hline Plantain, yellow, baked & Plátano amarillo al horno & Baked & 3 & 57.1 & 0.7 & $\begin{array}{c}9.4 \\
(10.3-9.0)\end{array}$ & $\begin{array}{c}2.2 \\
(2.5-1.8)\end{array}$ \\
\hline
\end{tabular}




\begin{tabular}{|c|c|c|c|c|c|c|c|}
\hline Potatoes, cream style & Papas en salsa blanca & Cream style & 3 & 81.3 & 2.4 & $\begin{array}{c}26.2 \\
(32.7-16.3)\end{array}$ & $\begin{array}{c}5.6 \\
(6.5-4.8)\end{array}$ \\
\hline Potatoes, fried & Papa frita & Fried & 3 & 63.6 & 3.3 & $\begin{array}{c}32.0 \\
(52.4-21.5)\end{array}$ & $\begin{array}{c}6.7 \\
(8.6-5.8)\end{array}$ \\
\hline Potatoes, mashed & Papa majada & Mashed & 3 & 74.0 & 2.3 & $\begin{array}{c}33.5 \\
(41.1-25.5)\end{array}$ & $\begin{array}{c}7.7 \\
(8.2-7.3)\end{array}$ \\
\hline Potato salad with eggs & $\begin{array}{l}\text { Ensalada de papas con } \\
\text { huevos }\end{array}$ & Boiled potatoes and eggs & 3 & 75.9 & 2.7 & $\begin{array}{c}65.1 \\
(83.4-45.8)\end{array}$ & $\begin{array}{c}7.6 \\
(8.3-6.9)\end{array}$ \\
\hline $\begin{array}{l}\text { Puerto Rican sweetpo- } \\
\text { tato, glazed }\end{array}$ & Batata mameya, glacé & Glazed & 3 & 41.8 & 1.0 & $\begin{array}{c}25.0 \\
(26.8-22.1)\end{array}$ & $\begin{array}{c}2.4 \\
(3.2-1.9)\end{array}$ \\
\hline Pumpkin, boiled & Calabaza hervida & Boiled & 3 & 86.6 & 0.9 & $\begin{array}{c}6.8 \\
(7.4-6.2)\end{array}$ & $\begin{array}{c}1.5 \\
(1.5-1.4)\end{array}$ \\
\hline Pumpkin, mashed & Calabaza majada & Mashed & 3 & 86.4 & 1.2 & $\begin{array}{c}15.6 \\
(16.0-15.2)\end{array}$ & $\begin{array}{c}2.0 \\
(2.6-1.5)\end{array}$ \\
\hline Spinach, boiled & Espinaca hervida & Boiled; butter added & 3 & 91.7 & 2.1 & $\begin{array}{c}24.4 \\
(25.9-23.5)\end{array}$ & $\begin{array}{c}6.0 \\
(6.1-5.8)\end{array}$ \\
\hline $\begin{array}{l}\text { Sweetpotato, white, } \\
\text { boiled }\end{array}$ & Batata blanca hervida & Boiled & 3 & 61.3 & 0.8 & $\begin{array}{c}13.9 \\
(17.8-10.4)\end{array}$ & $\begin{array}{c}2.5 \\
(3.0-2.1)\end{array}$ \\
\hline Tanier, white, boiled & Yautia blanca hervida & do. & 3 & 67.5 & 2.2 & $\begin{array}{c}21.1 \\
(25.0-18.2)\end{array}$ & $\begin{array}{c}13.2 \\
(14.4-12.6)\end{array}$ \\
\hline Tanier, yellow, boiled & Yautía amarilla hervida & do. & $\mathbf{3}$ & 68.3 & 1.7 & $\begin{array}{c}17.4 \\
(20.3-15.5)\end{array}$ & $\begin{array}{c}21.6 \\
(23.6-20.4)\end{array}$ \\
\hline "Tostones" of plantain & Tostones de plátano & Fried & $\mathbf{3}$ & 32.0 & 1.4 & $\begin{array}{c}15.6 \\
(17.0-12.9)\end{array}$ & $\begin{array}{c}5.6 \\
(6.7-4.8)\end{array}$ \\
\hline Yam, white, boiled & Name hervido & Boiled & 3 & 70.5 & 1.5 & $\begin{array}{c}19.8 \\
(23.3-13.6)\end{array}$ & $\begin{array}{c}3.7 \\
(4.4-2.8)\end{array}$ \\
\hline Meats: & & & & & & & \\
\hline Beef steak, baked & Beef steak al horno & Baked & 3 & 63.4 & 21.7 & $\begin{array}{c}589.3 \\
(677.1-528.5)\end{array}$ & $\begin{array}{c}107.0 \\
(133.8-92.0)\end{array}$ \\
\hline Chicken, baked & Pollo al horno & do. & 3 & 65.3 & 19.2 & $\begin{array}{c}444.9 \\
(576.4-325.8)\end{array}$ & $\begin{array}{c}83.1 \\
(109.8-66.7)\end{array}$ \\
\hline
\end{tabular}


TABLE 1.-Continued

\begin{tabular}{|c|c|c|c|c|c|c|c|}
\hline Name of dish in English & Name of dish in Spanish & Method of cooking & $\begin{array}{c}\text { Samples } \\
\text { tested }\end{array}$ & Moisture & $\begin{array}{c}\text { Protein, } \\
\text { wet } \\
\text { basis }\end{array}$ & $\begin{array}{c}\text { Methionine per 100 } \\
\text { gm. as served: } \\
\text { Average maximum } \\
\text { and minimum in } \\
\text { parentheses }\end{array}$ & $\begin{array}{l}\text { Cystine per } 100 \\
\text { gm. as scrved: } \\
\text { Averagemaximum } \\
\text { and minimum in } \\
\text { parentheses }\end{array}$ \\
\hline & & & Number & Percent & Percent & Milligrams & Afilligranes \\
\hline Entrails of chicken & Menudos de pollo & Stewed & 3 & 67.7 & 13.8 & $\begin{array}{c}251.9 \\
(312.3-132.8)\end{array}$ & $\begin{array}{c}55.7 \\
(63.1-51.6)\end{array}$ \\
\hline Goat, fricassee & Cabro en fricasé & do. & 3 & 63.7 & 22.0 & $\begin{array}{c}495.9 \\
(619.3-388.1)\end{array}$ & $\begin{array}{c}112.7 \\
(150.0-70.7)\end{array}$ \\
\hline Goat, stewed & Cabro estofado & do. & 3 & 59.1 & 27.3 & $\begin{array}{c}829.0 \\
(907.6-777.1)\end{array}$ & $\begin{array}{c}125.8 \\
(155.3-93.4)\end{array}$ \\
\hline Hamburger & Hamburguesa & Fried & 3 & 46.5 & 17.8 & $\begin{array}{c}430.1 \\
(453.7-385.7)\end{array}$ & $\begin{array}{c}148.5 \\
(169.0-111.7)\end{array}$ \\
\hline Hot dogs, stuffed & "Hot dogs" rellenos & $\begin{array}{l}\text { Stuffed with cheese and ba- } \\
\text { con }\end{array}$ & 3 & 42.8 & 20.8 & $\begin{array}{c}395.3 \\
(413.6-362.6)\end{array}$ & $\begin{array}{c}100.0 \\
(110.7-91.7)\end{array}$ \\
\hline Ham in wine & Jamón en vino & In wine sauce & 3 & 44.8 & 14.3 & $\begin{array}{c}319.1 \\
(396.9-277.1)\end{array}$ & $\begin{array}{c}65.4 \\
(75.1-59.7)\end{array}$ \\
\hline Meat, breaded & Carne empanada & Fried & 3 & 49.9 & 19.5 & $\begin{array}{c}512.0 \\
(587.7-461.4)\end{array}$ & $\begin{array}{c}104.7 \\
(115.4-96.8)\end{array}$ \\
\hline Meat, stuffed & Carne mechada & Stewed & 3 & 53.2 & 32.8 & $\begin{array}{c}876.1 \\
(998.7-631.8)\end{array}$ & $\begin{array}{c}191.0 \\
(251.6-121.3)\end{array}$ \\
\hline Meat, stewed & Carne guisada & do. & 3 & 63.7 & 17.1 & $\begin{array}{c}334.3 \\
(418.9-236.7)\end{array}$ & $\begin{array}{c}81.1 \\
(96.4-53.7)\end{array}$ \\
\hline Pork, thigh & Pernil de cerdo & Roasted & 3 & 51.2 & 30.2 & $\begin{array}{c}778.8 \\
(854.0-659.3)\end{array}$ & $\begin{array}{c}291.0 \\
(327.7-226.4)\end{array}$ \\
\hline Veal stew & Ternera estofada & Stewed & 3 & 72.1 & 16.0 & $\begin{array}{c}375.0 \\
(420.5-343.2)\end{array}$ & $\begin{array}{c}87.1 \\
(95.6-82.4)\end{array}$ \\
\hline Veal roast & Ternera al hotno & Roasted & 3 & 67.5 & 26.1 & $\begin{array}{c}796.9 \\
(930.5-541.8)\end{array}$ & $\begin{array}{c}154.4 \\
(176.9-114.8)\end{array}$ \\
\hline
\end{tabular}




\begin{tabular}{|c|c|c|c|c|c|c|c|}
\hline \multirow{2}{*}{$\begin{array}{l}\text { Meat mixtures: } \\
\text { "Asopao" of chicken }\end{array}$} & \multirow[b]{2}{*}{ Asopao de pollo } & \multirow{4}{*}{$\begin{array}{l}\text { Condiment slightly fried; } \\
\text { chicken, rice and tomato } \\
\text { sauce added, cooked until } \\
\text { done but soupy } \\
\text { Boiled chicken, eggs and } \\
\text { potatoes } \\
\text { Stewed }\end{array}$} & \multirow{3}{*}{$\begin{array}{l}3 \\
3\end{array}$} & \multirow{3}{*}{$\begin{array}{l}84.0 \\
72.4\end{array}$} & \multirow{3}{*}{$\begin{array}{l}4.5 \\
6.4\end{array}$} & \multirow[b]{2}{*}{$\begin{array}{c}124.3 \\
(167.8-102.2)\end{array}$} & \multirow[b]{2}{*}{$\begin{array}{c}17.2 \\
(20.5-14.4)\end{array}$} \\
\hline & & & & & & & \\
\hline Chicken salad & Ensalada de pollo & & & & & $\begin{array}{c}131.4 \\
(185.5-94.1)\end{array}$ & $\begin{array}{c}26.0 \\
(35.5-14.1)\end{array}$ \\
\hline Chili con carne & Chile con carne & & 3 & 71.5 & 7.5 & $\begin{array}{c}120.0 \\
(160.7-74.1)\end{array}$ & $\begin{array}{c}23.6 \\
(28.1-19.3)\end{array}$ \\
\hline "Hayacas" & Hayacas & Boiled & 3 & 69.8 & 6.8 & $\begin{array}{c}166.4 \\
(180.9-147.1)\end{array}$ & $\begin{array}{c}38.0 \\
(39.0-36.3)\end{array}$ \\
\hline "Pasteles" & Pasteles & do. & 3 & 70.0 & 5.1 & $\begin{array}{c}98.1 \\
(125.4-65.4)\end{array}$ & $\begin{array}{c}19.6 \\
(21.3-14.9)\end{array}$ \\
\hline "Pastelón de yautía" & Pastelón de yautía & Baked & 3 & 65.1 & 7.7 & $\begin{array}{c}167.9 \\
(203.8-116.2)\end{array}$ & $\begin{array}{c}51.3 \\
(52.9-49.6)\end{array}$ \\
\hline Rice with chicken & Arroz con pollo & $\begin{array}{l}\text { The same as "Asopao" of } \\
\text { chicken but cooked until } \\
\text { all water evaporates. }\end{array}$ & 3 & 48.5 & 12.9 & $\begin{array}{c}258.0 \\
(330.6-211.2)\end{array}$ & $\begin{array}{c}35.6 \\
(36.6-34.6)\end{array}$ \\
\hline Turkey chow mein & Chow mein de pavo & Stewed & 3 & 71.0 & 7.6 & $\begin{array}{c}140.4 \\
(166.2-124.7)\end{array}$ & $\begin{array}{c}38.3 \\
(44.8-34.3)\end{array}$ \\
\hline $\begin{array}{l}\text { Sea Foods: } \\
\text { Fish, breaded }\end{array}$ & Pescado empanado & Fried & 3 & 46.0 & 24.7 & $\begin{array}{c}706.3 \\
(726.8-669.6)\end{array}$ & $\begin{array}{c}87.0 \\
(90.9-80.3)\end{array}$ \\
\hline Lobster salad & Ensalada de langosta & $\begin{array}{l}\text { Lobster, potatoes and eggs } \\
\text { are cooked; other ingre- } \\
\text { dients are as available }\end{array}$ & 3 & 73.7 & 11.0 & $\begin{array}{c}297.5 \\
(386.3-226.4)\end{array}$ & $\begin{array}{c}62.2 \\
(85.7-46.5)\end{array}$ \\
\hline $\begin{array}{l}\text { Cereals: } \\
\text { "Asopao" of pigeonpeas }\end{array}$ & Asopao de gandules & $\begin{array}{l}\text { The same as "asopao" of } \\
\text { chicken but without } \\
\text { chicken and with pigeon- } \\
\text { peas }\end{array}$ & 3 & 86.4 & 1.6 & $\begin{array}{c}24.3 \\
(26.9-19.3)\end{array}$ & $\begin{array}{c}5.2 \\
(6.1-4.4)\end{array}$ \\
\hline
\end{tabular}


Table 1.-Concluded

\begin{tabular}{|c|c|c|c|c|c|c|c|}
\hline Name of dish in English & Name of dish in Spanish & Method of cooking & $\mid \begin{array}{c}S \text { amples } \\
\text { tested }\end{array}$ & Moisture & $\begin{array}{c}\text { Protein, } \\
\text { wet } \\
\text { basis }\end{array}$ & $\begin{array}{c}\text { Methionine per 100 } \\
\text { gm, as served: } \\
\text { Average maximum } \\
\text { and minimum in } \\
\text { parentheses }\end{array}$ & $\begin{array}{l}\text { Cystine per } 100 \\
\text { gm. as served: } \\
\text { Average maximum } \\
\text { and minimum in } \\
\text { parentheses }\end{array}$ \\
\hline & & & Number & Percent & Percent & Mfilligrams & Milligrams \\
\hline Bread, French & Pan francés & Baked & 3 & 26.1 & 11.4 & 172.9 & 143.4 \\
\hline Bread, sandwich & Pan especial & do. & 3 & 33.5 & 9.1 & 152.3 & 124.5 \\
\hline Rice, white & Arroz blanco & $\begin{array}{l}\text { Rice cooked with lard, salt, } \\
\text { and water until water } \\
\text { evaporates }\end{array}$ & 3 & 47.5 & 2.7 & $\begin{array}{c}64.6 \\
(66.7-63.0)\end{array}$ & $\begin{array}{c}17.2 \\
(18.4-16.3)\end{array}$ \\
\hline Rice with pigeonpeas & Arroz con gandules & $\begin{array}{l}\text { The same as rice, white, but } \\
\text { with pigeonpeas }\end{array}$ & 4 & 60.2 & 3.1 & $\begin{array}{c}50.5 \\
(54.9-48.2)\end{array}$ & $\begin{array}{c}16.2 \\
(19.0-15.0)\end{array}$ \\
\hline Spaghetti with pork & Spaghetti con cerdo & $\begin{array}{l}\text { Spaghetti boiled; sauce } \\
\text { contains ground pork } \\
\text { meat, tomato and other } \\
\text { ingredients; water added, } \\
\text { cooked until sauce thick- } \\
\text { ens }\end{array}$ & 3 & 69.8 & 5.2 & $\begin{array}{c}85.2 \\
(108.4-55.9)\end{array}$ & $\begin{array}{c}28.9 \\
(32.4-25.8)\end{array}$ \\
\hline Beans: & & & & & & & \\
\hline Chick peas & Garbanzos & Stewed & 3 & 77.4 & 3.7 & $\begin{array}{c}44.7 \\
(53.8-42.9)\end{array}$ & $\begin{array}{c}13.8 \\
(15.5-10.6)\end{array}$ \\
\hline Navy beans & Habichuelas blancas & do. & 3 & 79.8 & 3.8 & $\begin{array}{c}36.8 \\
(44.6-21.2)\end{array}$ & $\begin{array}{c}8.3 \\
(10.5-6.8)\end{array}$ \\
\hline Pigeonpeas & Gandules & do. & 3 & 77.9 & 3.3 & $\begin{array}{c}39.8 \\
(46.6-36.0)\end{array}$ & $\begin{array}{c}9.1 \\
(10.2-8.0)\end{array}$ \\
\hline Red kidney beans & Habichuelas coloradas & do. & 3 & 79.5 & 3.6 & $\begin{array}{c}38.5 \\
(44.5-33.8)\end{array}$ & $\begin{array}{c}7.4 \\
(7.9-7.2)\end{array}$ \\
\hline
\end{tabular}




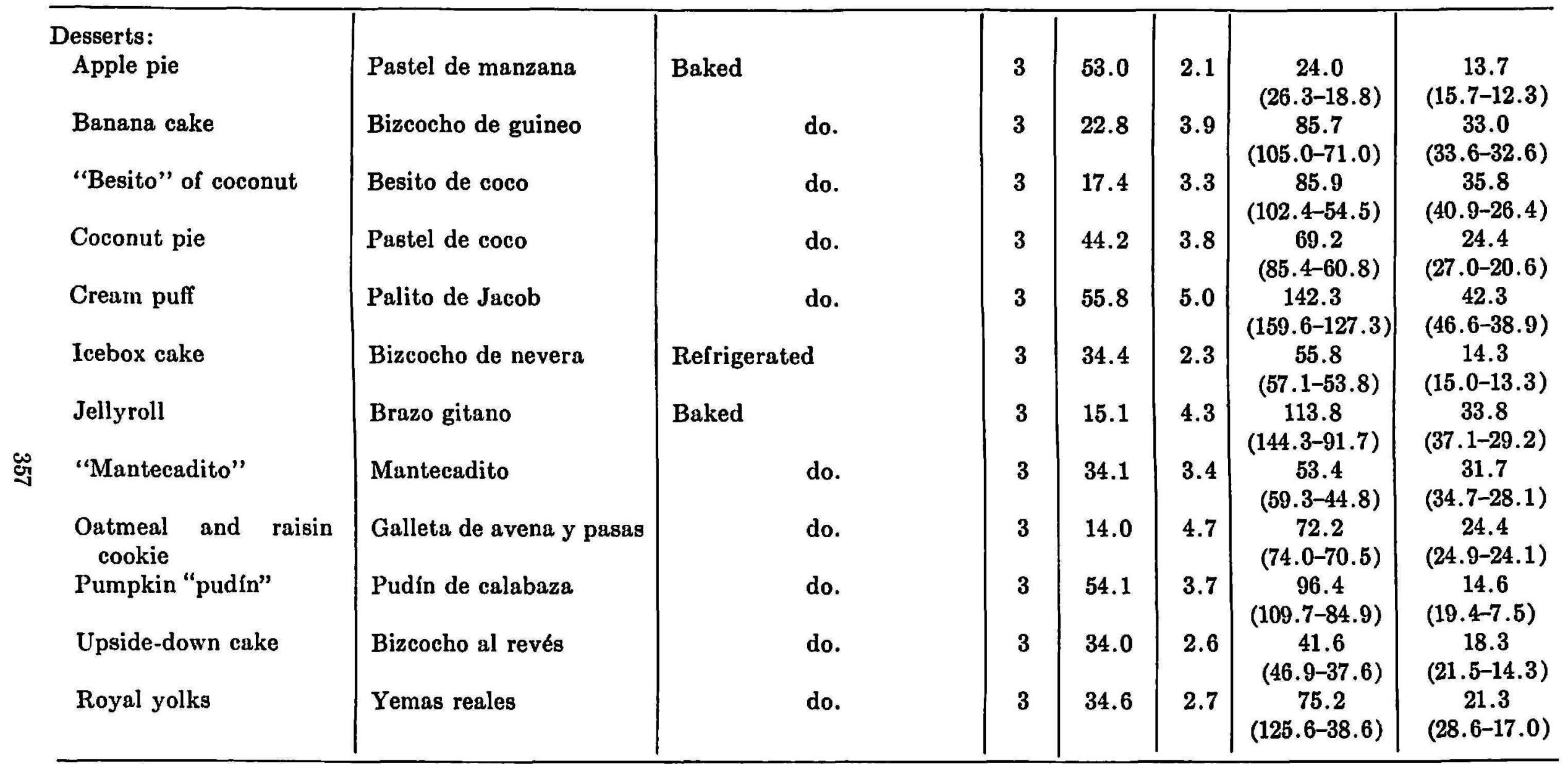


tained considerable egg, milk, and flour, were cream puff and jellyroll with 142.3 and $113.8 \mathrm{mg}$. per $100 \mathrm{gm}$. of methionine and 42.3 and 33.8 of cystine mg. per $100 \mathrm{gm}$., respectively.

On a cystine-free diet Rose (9) set $2.2 \mathrm{gm}$. of methionine per day as the definite safe value. In order to determine the nutritional adequacy of any food for methionine one must know its cystine content as, according to Rose (10), 80 to 89 percent of the methionine requirement in man can be met by cystine.

As the School Cafeteria serves lunches only, we have calculated out the methionine and cystine contents of two typical lunches. One lunch consisted of standard servings of a vegetable, meat, cereal, and salad dish, plus a dessert, but with the dish in each one of these categories having the lowest methionine-cystine content. At the other extreme we used a lunch menu comprising dishes in each of the above categories containing the highest content of methionine-cystine. The low methionine-cystine lunch menu was as follows: Sandwich bread, boiled arracacha, stewed entrails of chicken, "asopao" of pigeonpeas, carrot and raisin salad, and apple pie. The standard servings contributed $0.66 \mathrm{gm}$. of methionine and $0.15 \mathrm{gm}$. of cystine, that is, a total of $0.81 \mathrm{gm}$. of methionine-cystine. The high methionine-cystine luncheon consisted of the following dishes: French bread, whole-kernel corn, stuffed meat, rice with chicken, and cream puff. The standard servings contributed $1.61 \mathrm{gm}$. of methionine and $0.35 \mathrm{gm}$. of cystine, that is, $1.96 \mathrm{gm}$. of methionine-cystine. Therefore, both menus supplied the recommended intake of about $0.4 \mathrm{gm}$. of methionine per day that cannot be replaced with cystine. However, neither one provided in its entirety the remaining $1.8 \mathrm{gm}$., either as methionine or as cystine. According to these estimates, it can be stated that any lunch menu served in the cafeteria, if fully consumed, supplies anywhere between 36 and 90 percent of the safe recommended intake of methionine-cystine, even though beverages were not included.

\section{SUMMARY}

The methionine and cystine contents of 71 different dishes frequently served at lunch in the Cafeteria of the School of Medicine of the University of Puerto Rico was determined. It was found that these lunches, if fully consumed, could supply anywhere between 36 and 90 percent of the daily recommended allowance of methionine-cystine.

\section{RESUMEN}

Se determinó el contenido de metionina y cistina en los 71 platos servidos con mayor frecuencia a la hora del almuerzo en la Cafetería de la Escuela de Medicina de la Universidad de Puerto Rico. Se encontró que un al- 
muerzo completo compuesto por estos manjares, si se consume todo, puede suministrar entre el 36 y el 90 porciento del requerimiento diario de metionina-cistina.

\section{LITERATURE CITED}

1. Cook, D. H., Axtmayer, J. H. and Dalmau, L. M., Nutrition studies of foodstuffs used in the Puerto Rican dietary, VII, A comparative study of the nutritive value of 3 diets of frequent use in Puerto Rico, P.R.J. Pub. Health \& Trop. Med. $163-13,1940$.

2. Axtmayer, J. H., Apuntes sobre nutrición, VI, El valor nutritivo de las proteínas, El Crisol, 1 3-8, 1947.

3. Asenjo, C. F., and Goyco, J. A., Effect of supplementing polished rice and red kidney beans diet with amino acids, Fed. Am. Biol. Soc. Proc. 13 450, 1954.

4. Goyco, J. A., Nitrogen balance of young adults consuming a deficient diet supplemented with Torula yeast and other nitrogenous products, $J$. Nulrn. 69 49-57, 1959.

5. Official Methods of Analysis of the Association of Official Agricultural Chemists, 8th ed., 12, Washington, D.C., 1955.

6. Stokes, J. L., Gunnes, M., Dwyer, I. M., and Caswell, M. C., Microbiological methods for the determination of amino acids, II, A uniform assay for the 10 essential amino acids, J. Biol. Chem., $16035-49,1945$.

7. Barton-Wright, E. C., Microbiological Assay of the Vitamin B Complex and Amino Acids, Pitman Publishing Co., New York, N.Y., 142-6, 1952.

8. Horn, M. J. and Blum, A. E., A microbiological method for determination of cystine in foods, Cereal Chem., 33 (1) 18-28, 1956.

9. Rose, W. C., Amino acids requirement of man, Fed. Am. Biol. Soc. Proc. 8 546-52, 1949.

10. Rose, W. C., and Wixom, R. L., The amino acid requirements of man, XIII, The sparing effect of cystine on the methionine requirement, $J$. Biol. Chem. 216 763-73, 1955. 